\title{
A Flexible Presentation Tool for Diverse Multi-display Environments
}

\author{
Kazutaka Kurihara $^{1}$ and Takeo Igarashi ${ }^{2}$ \\ ${ }^{1}$ National Institute of Advanced Industrial Science and Technology, \\ Akihabara Daibiru 10F, 1-18-13 Sotokanda, Chiyoda-ku, Tokyo, 101-0021, Japan \\ ${ }^{2}$ Department of Computer Science, The University of Tokyo, \\ 7-3-1 Hongo, Bunkyo-ku, Tokyo, 113-0033, Japan \\ k-kurihara@aist.go.jp, takeo@acm.org
}

\begin{abstract}
Display environments for presentations are becoming diverse, and it is common to have multiple displays in the same room. We present a system to edit and give presentations using multiple displays. The main idea is to separate the content from the views. The author prepares presentation visuals (text and images) on an infinitely zoomable canvas, and then specifies what is shown in each display by dragging a rectangular display proxy onto the canvas. The presenter can change what is shown in each display by manipulating these display proxies. We describe an example that showed that the system can handle various presentation scenarios using multiple displays.
\end{abstract}

\section{Introduction}

Presentation environments are becoming increasingly diverse, and it is not uncommon for presentation rooms to have multiple displays. In theory, a presenter could use a huge number of displays simultaneously, including the personal laptops in the audience. However, current presentation tools are not designed to take advantage of such a situation. Most standard presentation tools are designed primarily for singledisplay presentations, but do support additional control views that are automatically created for the presenter.

Chiu et al. [1] and Zhang et al. [4] proposed "slide-based" multi-display presentation systems. The user can paste slides onto a multi-track timeline that corresponds to these displays, or show the slides on multiple displays successively in an order predefined by a special script language. These methods are effective for users whose presentation environments do not frequently change and who carefully prepare the presentation; for example, in a business where presentations are given in a fixed meeting room, often following a prepared script with little deviation.

One drawback of this approach is that it is somewhat difficult to "adjust" a presentation to different multi-display configurations. The user must align the displays using the script language, or rewrite individual slides. If multiple displays are used to show a large image, the user must divide the image into sub-views for each display. In addition, this approach, due to its fixed structure, is not flexible enough to support on-the-fly improvisation, such as showing a global overview or adding 
discussion side notes. These online improvisations are particularly important in an educational environment, because interaction is essential for learning.

We propose a new presentation system that makes it easier to create and show presentations using multiple displays. The main idea is to separate the content from the views in order to adapt flexibly to a diverse array of display settings.
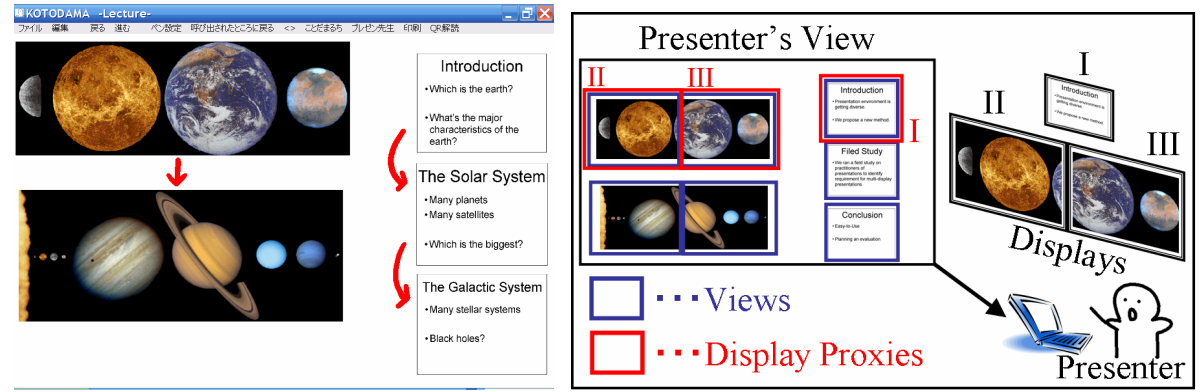

Fig. 1. System overview. (Left) Snapshot of multi-display presentation material on the editing canvas. Note that some information is presented in unfamiliar aspect ratios. (Right) Display proxy objects and view objects.

\section{System Description}

The system is based on the metaphor of an infinitely zoomable canvas [2, 3]. The user can freely navigate the canvas by panning and zooming, and can place presentation visuals (text and images) anywhere on the canvas (Fig. 1, left). To show visuals on a display, the user simply associates a view with a display proxy (Fig. 1 right, red), by placing the proxy object on top of the view. After the presentation visuals are prepared, the user can define a sequence of views to define the basic flow of visuals to the audience, navigated by simple button operations. To do so, the user simply presses the "capture" button to create and register a rectangular view object (Fig. 1 right, blue) that encloses the current content in the display proxy.

Importantly, the editing of visuals is separated from the presentation views. This is very important when a presenter must give the same presentation in various display settings. In traditional slide-based presentation tools (including Chiu et al. and Zhang et al.), visuals are tightly coupled with views, so it is very difficult to show the same material in different display settings. To adapt such presentations to a new setting may require making new slides from scratch. However, using our method, the user needs only to change the views; the presentation visuals remain unchanged.

The capability of continuous view control on the infinite canvas is also helpful for improvising using multiple displays. Display proxies on the canvas allow the user to quickly specify what to show in each display. When the presenter wants to discuss a topic in further detail, he can slightly zoom out or pan to a vacant space in the working display without affecting other displays, and can also quickly show a global 
overview by zooming out. The lasso grouping function to view proxy objects helps the user to move multiple view proxies synchronously.

Defining views on a canvas is not a new concept $[2,3]$; our contribution is using them to control multiple displays and proposing methods to coordinate them. We assume that the system knows which displays are available, how to establish connections with them, and how to synchronize the presentation visuals in a display proxy and the actual view on the corresponding display. A solution for such lowerlevel issues will be addressed in a future paper.

\section{Experiences}

In this section, we describe the operation of various presentation scenarios using our prototype system.

Using 3+1 adjacent displays. We aligned three displays of the same size horizontally, and put one relatively small display below the right display (Fig. 2). The presenter showed various paintings with unfamiliar aspect ratios. The three main displays showed the paintings, while the relatively small one displayed some comments on the paintings.

Showing successive slides at a time. We tested a scenario where a presenter with a presentation designed for a single-display environment wants to show it using multiple displays (Fig. 3). We assumed that the presenter prepared a slide-based presentation in advance by arranging the slides horizontally. However, as it turned out that he could use three displays, he decided to show the previous, current, and next slides simultaneously to facilitate learning.
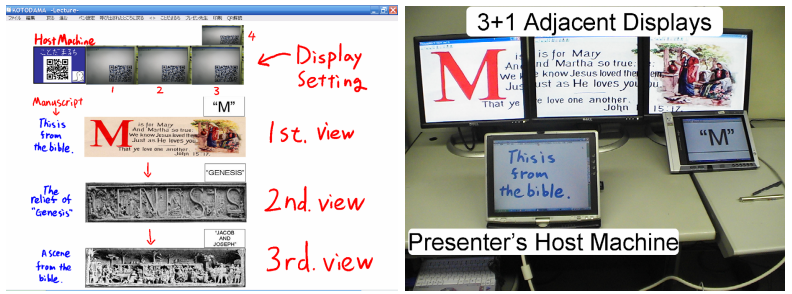

Fig. 2. Using $3+1$ adjacent displays. (Left) Presentation material on the editing canvas. (Right) Snapshot of the environment.
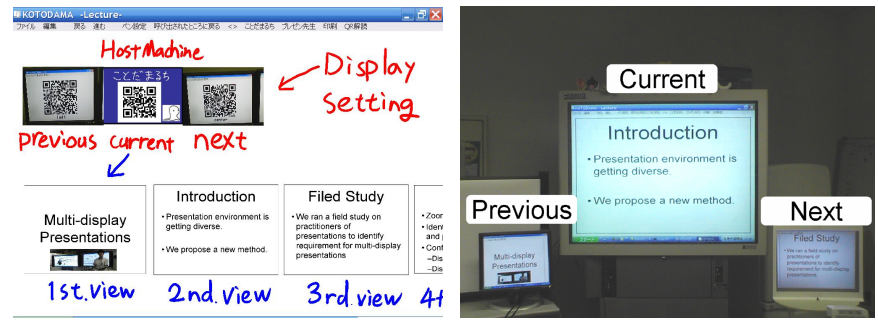

Fig. 3. Showing three successive slides simultaneously. (Left) Presentation material on the editing canvas. (Right) Snapshot of the environment. 

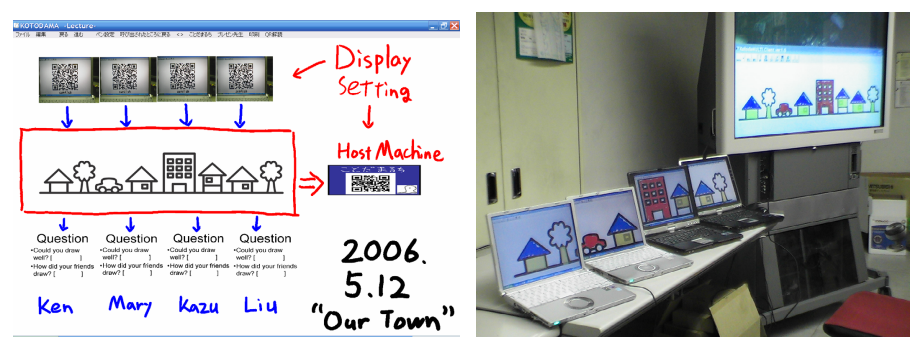

Fig. 4. Collaborative painting. (Left) Presentation material on the editing canvas. (Right) Snapshot of the environment.

Collaborative painting. We assumed that the presenter wanted students to work on a collaborative painting (Fig. 4). Each student (the audience) could work on a portion of the painting on an individual computer, while the main display in the room showed the overview of the whole painting. After the project was complete, the presenter distributed questionnaires to each student.

\section{Conclusion and Future Work}

We presented a prototype presentation tool that separates content from views to flexibly adapt to diverse display settings. It seamlessly extends user-working styles in ordinary single-display presentations to multi-display environments. We also described various presentation scenarios using our prototype system, which demonstrated its versatility and effectiveness.

We are planning to run a formal user study to evaluate and improve our system. Another direction of future work is to establish a method for editing presentations for non-rectangular displays by developing interfaces that allow non-rectangular view objects, enabling presentations for even more diverse display environments.

Acknowledgments. This research was partially supported by Microsoft Institute for Academic Research Collaboration (IJARC) Core2 Project and grant from MSRA Mobile Computing in Education Theme program.

\section{References}

1. Chiu, et al.: Manipulating and Annotating Slides in a Multi-Display Environment. In: INTERACT'03, pp. 583-590 (2003)

2. Good, et al.: CounterPoint: Creating Jazzy Interactive Presentations. HCIL Tech Report \#2001-03, University of Maryland (2001)

3. Perlin, et al.: An Alternative Approach to the Computer Interface. In: SIGGRAPH'93, pp. 57-64 (1993)

4. Zhang, et al.: A Presentation Authoring Tool for Media Devices Distributed Environment. In: ICME'04 (2004) 